\title{
Quantités d'herbe ingérées par les vaches laitières, les génisses et les moutons : effet de quelques facteurs de variation et comparaison entre ces types d'animaux
}

\author{
J.P. Dulphy ${ }^{1}$, C. Demarquilly ${ }^{1}$ et B. Rémond 2
}

avec la collaboration technique de L. L'Hotelier ${ }^{1}$, A. Ollier ${ }^{2}$, P. D'Hour ${ }^{3}$, J.M. Giraud ${ }^{4}$, J. Bony 5 et J. Rouel 5

1 Station de recherches sur la nutrition des herbivores,

2 Laboratoire de la lactation,

${ }^{3}$ Domaine de Marcenat,

${ }^{4}$ Domaine de Laqueuille,

5 Domaine d'Orcival, INRA, Centre de recherches de Clermont-Ferrand, Theix, 63122 Ceyrat, France

(reçu le 23 novembre 1988, accepté le 3 juin 1989)

Résumé - Afin de mieux connaître les quantités d'herbe de référence (dans le système français des unités d'encombrement) ingérées par des bovins de type très différent et les liaisons entre les quantités d'herbe ingérées par des ovins et des bovins, nous avons, en mai, juin et juillet, mesuré les quantités ingérées d'herbe coupée chaque jour et distribuée d'une part à des ovins, d'autre part soit à des vaches laitières, soit à des génisses de race laitière, soit à des génisses de race à viande (Charolaise et Limousine). Un échantillon de fourrage vert correspond à tout ce qui a été fauché et distribué pendant une semaine. Nous disposons alors de :

- 26 comparaisons moutons - vaches laitières;

- 25 comparaisons moutons - génisses de race laitière;

- 17 comparaisons moutons - génisses de race à viande.

Dans un premier temps, nous avons analysé les facteurs de variation liés à la plante. Ainsi les quantités ingérées d'herbe ont-elles été, en moyenne, très comparables entre le premier et le second cycle de végétation. Au premier cycle, elles ont diminué nettement d'une semaine à l'autre. Globalement, l'effet de la teneur en MS n'a pas été significatif dans le cadre de ces observations; l'effet de la digestibilité n'a été significatif que sur les quantités ingérées par les génisses de race à viande. Si on retire les effets du poids vif pour tous les animaux et de la production de lait pour les vaches, les coefficients de variation des quantités ingérées intralot sont égaux à : $6,6 \%$ pour les vaches laitières, $9,1 \%$ pour les génisses de race laitière, $7,9 \%$ pour les génisses de race à viande. Les relations entre les quantités d'herbe ingérées, exprimées en g de MS $/ \mathrm{kg} \mathrm{P}^{0.75}$, par les ovins (QIM) et les bovins sont les suivantes :

- vaches laitières : $\mathrm{QIVL}=33,5+0,933 \mathrm{QIM}+1,58 \mathrm{PL} 4$

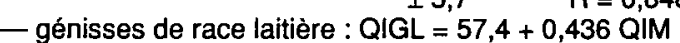

$$
\begin{aligned}
& \pm 5,7 \quad R=0,848 \\
& L=57,4+0,436 \text { QIM }
\end{aligned}
$$

— génisses de race à viande: $\mathrm{QIGV}=37,0+0,559 \mathrm{QIM}$

$$
\pm 7,2 \quad R=0,530 \text {. }
$$

Compte tenu de ces résultats, les quantités ingérées de l'herbe de référence sont les suivantes : $75 \mathrm{~g}$ de MS/kg $P^{0,75}$ pour les moutons (par convention), $143 \mathrm{~g}$ de MS $/ \mathrm{kg} \mathrm{P}^{0,75}$ pour une vache laitière de $600 \mathrm{~kg}$ produisant $25 \mathrm{~kg}$ de lait, $90 \mathrm{~g}$ de $\mathrm{MS} / \mathrm{kg} \mathrm{P}^{0,75}$ pour une génisse de race laitière pesant $400 \mathrm{~kg}, 79 \mathrm{~g}$ de MS/kg P ${ }^{0,75}$ pour une génisse de race à viande pesant $400 \mathrm{~kg}$.

ingestion - fourrage vert - vaches laitières - génisses - moutons 
Summary - Level of grass intake in dairy cows, heifers and sheep : effect of some factors of variation and comparison between types of animals. The level of intake of cut grass offered each day to sheep, dairy cows, dairy heifers and beef heifers (Charolais and Limousin) was measured in May, June and July in order to determine more accurately :

- amounts of grass ingested by very different types of cattle and used as a reference in the French system of fill units;

- relations between levels of grass intake in sheep and cattle.

The green forage sample used corresponded to the total amount of grass cut and fed over a period of one week. The study concerned:

-26 comparisons of sheep and dairy cows;

-25 comparisons of sheep and dairy heifers;

-17 comparisons of sheep and beef heifers.

The plant-related factors of variation were first examined : the level of grass intake was, on an average, very similar between the first and the second growth cycle. During the first cycle it decreased from one week to another; the effect of dry matter content was not significant during our observations and that of digestibility was only significant for amounts ingested by beef heifers.

If the effects of liveweight for all animals and of milk production for cows are subtracted the withingroup coefficients of variation of amounts ingested were : $-6.6 \%$ for dairy cows, $-9.1 \%$ for dairy heifers, $-7.9 \%$ for beef heifers. Levels of grass intake, expressed in $g$ of DM/kg $W^{0,75}$, in sheep (QIM) as related to cattle (QIVL, QIGL, QIGV) were the following:

dairy cows

$Q I V L=33.5+0.933 Q I M+1.58 P L 4$

dairy heifers

$$
\pm 5.7 \quad R=0.848
$$

$Q I G L=57.4+0.436$ QIM

beef heifers

$$
\pm 6.8 \quad R=0.472
$$

QIGV $=37.0+0.559$ QIM

$$
\pm 7.2 \quad R=0.530 \text {. }
$$

On account of these results, the levels of reference grass intake were the following :

- $75 \mathrm{~g} \mathrm{DM} / \mathrm{kg} W^{0,75}$ for sheep

- $143 \mathrm{~g} \mathrm{DM} / \mathrm{kg}$ W $^{0,75}$ for a $600 \mathrm{~kg}$ dairy cow yielding $25 \mathrm{~kg}$ milk

- $90 \mathrm{~g}$ DMM0,75 for a $400 \mathrm{~kg}$ dairy heifer

- $79 \mathrm{~g} \mathrm{DMM} 0.75$ for a $400 \mathrm{~kg}$ beef heifer

intake - green forage - dairy cows - heifers - sheep

\section{INTRODUCTION}

Par définition (Jarrige et al., 1986), $1 \mathrm{~kg}$ de matière sèche d'herbe au stade pâturage avec des caractéristiques bien précises (Jarrige, 1979) a une valeur d'encombrement égale à 1 . Cette valeur correspond à une ingestibilité de cette herbe, dite "de référence", égale à $75 \mathrm{~g}$ de $\mathrm{MS} / \mathrm{kg} \mathrm{P}^{0,75}$ chez le mouton et à $123 \mathrm{~g}$ chez la vache laitière pesant $600 \mathrm{~kg}$ et produisant $17 \mathrm{~kg}$ de lait. II existe par ailleurs chez le mouton des données très nombreuses concernant l'ingestibilité des fourrages verts (INRA, 1988). Des liaisons mathématiques peuvent être ensuite utilisées pour extrapoler à d'autres catégories de ruminants les résultats trouvés sur moutons.

Ainsi, les liaisons établies par Demarquilly \& Weiss (1971) concernaient 64 comparaisons entre moutons adultes et vaches laitières faibles productrices d'une part, et 58 comparaisons entre les moutons et bœufs de $450 \mathrm{~kg}$. Pour les grami- 
nées, 28 comparaisons concernant les vaches et 49 les bœufs donnaient les relations suivantes ( $Q$ l exprimées en g de MS/ $\mathrm{kg} \mathrm{P0,75)}$ :

QI VL $=54,1+0,807$ QIM $\pm 7,1 \quad R=0.824$

$\mathrm{Q}$ I $=48,2+0,801 \mathrm{QIM} \pm 9,6 \mathrm{R}=0,562$

Plus récemment, nous avons effectué 11 comparaisons entre moutons et génisses de race laitière âgés de 1 an (Dulphy \& Michalet-Doreau, 1983) et calculé la relation suivante :

QI $G=19,7+1,023$ QIM $\pm 5,3 \quad R=0,923$

A notre connaissance, il n'y a pas de données de ce type dans la littérature. Nous avons donc, en prenant toujours le mouton standard comme témoin, étudié les quantités de fourrage vert ingérées par des vaches laitières produisant environ 25 $\mathrm{kg}$ de lait au début des essais, des génisses de race laitière et des génisses de race à viande pesant 350 à $450 \mathrm{~kg}$. Des résultats préliminaires ont déjà été présentés (Dulphy \& D'Hour, 1987).

Notre objectif était double :

- mieux connaître les quantités ingérées de l'herbe de référence pour des bovins de type très différent;

- préciser et aussi compléter les liaisons, établies autrefois par Demarquilly \& Weiss (1971), entre les quantités ingérées par des ovins et des bovins, en étudiant des fourrages verts au cours de leur évolution.

L'ensemble des mesures présentées correspond aux essais mis en place en vue d'améliorer le système des UEB publié récemment (Dulphy et al., 1987).

\section{MATÉRIEL ET MÉTHODES}

Des mesures journalières individuelles ont été effectuées, en mai, juin et juillet, au cours de 2 années successives, sur différents lots d'ani- maux présents sur les domaines INRA de Theix (850 m d'altitude), Marcenat (1 $100 \mathrm{~m}$ ), Laqueuille $(1100 \mathrm{~m})$ et Orcival $(1000 \mathrm{~m})$. On appellera "échantillon" l'herbe distribuée pendant une semaine à une catégorie de bovins. Les caractéristiques générales de ces échantillons figurent dans le Tableau I.

\section{Fourrages}

Les fourrages étudiés, fauchés chaque matin, étaient distribués aux animaux. II s'agissait avant tout de fourrages de prairie naturelle de montagne au cours du premier et du second cycle de végétation, mais il y a eu également des repousses de ray-grass anglais (2 échantillons à Theix et 5 à Marcenat), des repousses de dactyle (3 échantillons à Orcival) et de la fléole au premier cycle (à Laqueuille). Les teneurs moyennes en matière sèche de ces fourrages et leur digestibilité, mesurée sur les moutons, sont données dans le Tableau II.

\section{Animaux et alimentation}

Dans chaque domaine, un lot de 6 moutons (béliers castrés de race Texel), pesant 60 à $70 \mathrm{~kg}$ et placés en cages à métabolisme, a été utilisé chaque année pour recevoir aussi les échantillons de fourrages destinés aux bovins.

En 1985 à Theix, Marcenat et Orcival, ainsi qu'en 1986 à Orcival, nous avons utilisé, dans chaque lieu, un lot de 9-10 vaches laitières de race FFPN $\times$ Holstein. Les animaux ont reçu systématiquement $1 \mathrm{~kg}$ de tourteau tanné (mélange de soja et de colza) par jour et du concentré équilibré pour la production de lait $(1,05$ UFU $\mathrm{kg}$ de MS et $122 \mathrm{~g}$ de PDI) afin de couvrir leurs besoins, surtout lorsque la valeur alimentaire de l'herbe baissait en fin de premier cycle. Au total, 14 échantillons concernent le premier cycle de végétation et 12 le second.

En 1985, à Marcenat et Orcival, en 1986, à Laqueuille et Orcival, nous avons utilisé aussi un lot de génisses ( 7 animaux à Marcenat et 10 ailleurs) de race laitière (Montbéliarde et FFPN x Holstein à Marcenat, FFPN $x$ Holstein ailleurs). 
Tableau I. Caractéristiques générales des échantillons étudiés ( 1 échantillon = 1 semaine de mesures).

\begin{tabular}{|c|c|c|c|c|c|c|c|}
\hline \multirow{2}{*}{$\begin{array}{l}\text { Lieu et année } \\
\text { Plante }\end{array}$} & \multirow[t]{2}{*}{ Cycle } & \multicolumn{2}{|c|}{ Vaches laitières } & \multicolumn{4}{|c|}{ Génisses } \\
\hline & & $A$ & $P$ & \multicolumn{2}{|c|}{ Pie Noires } & \multicolumn{2}{|c|}{ à viande } \\
\hline \multicolumn{8}{|l|}{ Orcival, 1985} \\
\hline $\begin{array}{l}\text { Prairie naturelle } \\
\text { Dactyle }\end{array}$ & $\begin{array}{l}1 \\
2 \\
2\end{array}$ & 10 & $\begin{array}{l}5 \\
1 \\
2\end{array}$ & 10 & $\begin{array}{l}5 \\
0 \\
2\end{array}$ & $9(\mathrm{CH})$ & $\begin{array}{l}6 \\
0 \\
2\end{array}$ \\
\hline \multicolumn{8}{|l|}{ Marcenat, 1985} \\
\hline $\begin{array}{l}\text { Prairie naturelle } \\
\text { Ray-grass anglais }\end{array}$ & $\begin{array}{l}1 \\
2\end{array}$ & 9 & $\begin{array}{l}4 \\
5\end{array}$ & 7 & $\begin{array}{l}4 \\
5\end{array}$ & $8(\mathrm{LI})$ & $\begin{array}{l}4 \\
5\end{array}$ \\
\hline \multicolumn{8}{|l|}{ Theix, 1985} \\
\hline $\begin{array}{l}\text { Prairie naturelle } \\
\text { Ray-grass anglais }\end{array}$ & $\begin{array}{l}1 \\
2 \\
2\end{array}$ & 9 & $\begin{array}{l}1 \\
2 \\
2\end{array}$ & & & & \\
\hline \multicolumn{8}{|l|}{ Orcival, 1986} \\
\hline $\begin{array}{l}\text { Prairie naturelle } \\
\text { Dactyle }\end{array}$ & $\begin{array}{l}1 \\
2\end{array}$ & 9 & 4 & 10 & $\begin{array}{l}4 \\
1\end{array}$ & & \\
\hline \multicolumn{8}{|l|}{ Laqueuille, 1986} \\
\hline Fléole & 1 & & & 10 & 4 & & \\
\hline Total & & & 26 & & 25 & & 17 \\
\hline
\end{tabular}

A : animaux/lot; $P$ : périodes de mesures.

Ces animaux pesaient en moyenne $370 \mathrm{~kg}$ en début d'essai et $417 \mathrm{~kg}$ à la fin, les 2 pesées étant espacées de 45 jours. Les génisses n'ont pas reçu d'aliment concentré. Au total, nous disposons de 25 échantillons, dont 17 pour le premier cycle.

Enfin, en 1985, nous avons utilisé un lot de 8 génisses de race Limousine à Marcenat et un lot de 9 génisses de race Charolaise à Orcival. Elles pesaient en moyenne $353 \mathrm{~kg}$ en début d'essai et $397 \mathrm{~kg}$ à la fin. Pour ces animaux, nous disposons de 17 échantillons, dont 10 pour le premier cycle.
Tous les fourrages ont été distribués à volonté (5 à 10\% de refus) aux animaux mis en comparaison. Les caractéristiques moyennes principales des animaux sont aussi données dans le Tableau II.

\section{Mesures}

Les quantités de matière verte et de matière sèche ingérées par chaque animal ont été mesurées chaque jour par pesée des quantités dis- 
Tableau II. Caractéristiques moyennes par lot et quantités d'herbe ingérées par semaine.

\begin{tabular}{lccc}
\hline & $\begin{array}{c}\text { Vaches laitières } \\
\text { Pie Noires }\end{array}$ & $\begin{array}{c}\text { Génisses } \\
\text { Pie Noires }\end{array}$ & $\begin{array}{c}\text { Génisses } \\
\text { à viande }\end{array}$ \\
\hline Nombre d'échantillons * & 26 & 25 & 17 \\
Poids vif moyen des animaux (kg) & $605 \pm 21$ & $392 \pm 18$ & $376 \pm 18$ \\
Production de lait à 4\% de MG (kg) & $21,0 \pm 2,8$ & - & - \\
Teneur en MS de l'herbe (\%) & $17,5 \pm 2,5$ & $19,1 \pm 4,2$ & $18,0 \pm 2,8$ \\
Digestibilité de la MO (\%) (mesurée sur moutons) & $69,2 \pm 3,4$ & $68,1 \pm 5,8$ & $69,1 \pm 3,8$ \\
Ingestibilité de l'herbe par les moutons (g MS/kg P0,75) & $73,3 \pm 7,7$ & $68,6 \pm 9,0$ & $69,9 \pm 6,3$ \\
$\begin{array}{l}\text { Quantités de MS ingérées par les bovins (g MS/kg P0,75) } \\
\text { herbe }\end{array}$ & $133,9 \pm 10,4$ & $87,5 \pm 8,9$ & $76,1 \pm 8,2$ \\
\hline
\end{tabular}

* Un échantillon d'herbe correspond à une semaine de mesures.

tribuées et refusées et détermination de la teneur en matière sèche du fourrage distribué et des refus journaliers ( 7 jours/semaine).

Les animaux ont été pesés toutes les semaines, sauf à Marcenat (tous les 14 jours).

La digestibilité de la matière organique des fourrages a été mesurée sur moutons par collecte totale des fèces durant 6 jours/semaine.

Nous appellerons QIM, QIVL, QIGL et QIGV les quantités journalières de MS ingérées (en g de MS/kg Po,75) respectivement par les moutons, les vaches, les génisses de race laitière et celles de race viande. De même, MS (en pourcentage), DMO (en pourcentage), PL4 (en kg/j de lait à $4 \%$ de matières grasses) et PV (en $\mathrm{kg}$ ) représenteront respectivement la teneur en matière sèche des fourrages, leur digestibilité, la production des vaches et le poids vif des animaux.

\section{Traitements statistiques}

Les analyses statistiques appliquées aux résultats obtenus sont tout à fait classiques : comparaisons de moyennes, régressions simples ou multiples, variabilités intralot.

\section{RÉSULTATS}

\section{Présentation de la base de données}

\section{Quantités ingérées moyennes}

\section{Vaches laitières}

Les vaches ont ingéré en MS/jour, respectivement $15,78,1,04$ et $0,87 \mathrm{~kg}$ de fourrage vert, de concentré équilibré et de tourteau tanné. Toutes les vaches ayant reçu le tourteau, nous n'en avons pas tenu compte dans le dépouillement des données, d'autant plus que sa valeur d'encombrement doit être négligeable (digestion rapide). En revanche, nous avons considéré que le taux de substitution fourrage/ concentré était de 0,5 (valeur choisie d'après les résultats de Sarker \& Holmes, 1974; Meijs, 1981; Meijs \& Hoekstra, 1984; Stakelum, 1986a et b) et calculé une quan- 
tité théorique d'herbe ingérée (QIVL) égale à la quantité réelle additionnée de la quantité de concentré équilibré multipliée par 0,5 .

Dans ces conditions, la quantité théorique de fourrage ingérée en l'absence d'aliment concentré est égale à $16,3 \mathrm{~kg}$ de MS (Tableau III), soit 2,69 kg par $100 \mathrm{~kg}$ de poids vif, soit $134 \mathrm{~g} / \mathrm{kg} P^{0.75}$.

\section{Génisses}

Les génisses n'ayant pas reçu d'aliment concentré, il n'y a pas à faire d'hypothèse sur le taux de substitution fourrage/ concentré.

Les génisses de race laitière ont ingéré $7,7 \mathrm{~kg}$ de MS/jour, soit 1,96 kg par $100 \mathrm{~kg}$ de poids vif, soit $87,5 \mathrm{~g} / \mathrm{kg} \mathrm{P}^{0,75}$. Quant aux génisses de race à viande, elles ont ingéré nettement moins, $6,5 \mathrm{~kg}$ de $\mathrm{MS} /$ jour, soit $1,73 \mathrm{~kg}$ par $100 \mathrm{~kg}$ de poids vif, soit $76 \mathrm{~g} / \mathrm{kg} \mathrm{P}^{0,75}$.

D'ailleurs, pour les 17 fourrages verts distribués simultanément aux 2 types d'animaux, les quantités ingérées par les génisses de race laitière sont significative- ment supérieures à celles ingérées par les génisses de race à viande $: 86,8 \mathrm{~g}$ de $\mathrm{MS} /$ kg $\mathrm{P}^{0,75}$ contre 76,1 .

\section{Facteurs de variation liés à la plante}

\section{Cycle de végétation}

Les quantités de matière sèche d'herbe ingérées ont été, en moyenne, très proches, voire identiques, entre le premier et le second cycle de végétation :

- 133,9 g de MS/kg P0,75 dans les 2 cas pour les vaches;

- 85,8 contre $90,7 \mathrm{~g}$ de MS/kg $\mathrm{P}^{0,75}$ pour les génisses de race laitière;

- 73,6 contre $79,7 \mathrm{~g}$ de MS/kg $\mathrm{P}^{0,75}$ pour les génisses de race à viande.

\section{Stade de végétation}

Au premier cycle de végétation, il y a eu une évolution caractéristique des quantités d'herbe ingérées : augmentation entre la première et la deuxième et parfois jusqu'en troisième semaine, puis diminu-

Tableau III. Moyennes et variabilités intra lot des quantités ingérées d'herbe.

\begin{tabular}{lccc}
\hline & \multicolumn{2}{c}{ Race laitière } & $\begin{array}{c}\text { Race à viande } \\
17 \text { génisses }\end{array}$ \\
\hline (en kg de MS/animal/jour) & & & \\
Moyenne & $16,3^{*}$ & 7,7 & 6,5 \\
Ecart type initial & $\pm 1,68$ & $\pm 1,02$ & $\pm 0,95$ \\
Effet PL4 (QI/PL4) & 0,139 (NS) & - & - \\
Effet PV (QI/PV)** & 0,693 (NS) & $2,22(\mathrm{~S})$ & $1,37(\mathrm{~S})$ \\
Ecart type final & $\pm 1,08(6,6 \%)$ & $\pm 0,73(9,5 \%)$ & $\pm 0,54(8,3 \%)$ \\
\hline
\end{tabular}

- Quantités ingérée d'herbe $+50 \%$ de la quantité de concentré équilibré; "* pente de la régression. 
tion rapide. Les valeurs observées ont été les suivantes :

$-+10 \mathrm{~g}$ de $\mathrm{MS} / \mathrm{kg} \mathrm{P}^{0,75}$ sur une semaine 2 fois, puis chute de $7,3 \mathrm{~g} /$ semaine durant les 3 à 4 semaines d'étude;

- + 4,5 g de MS/kg P0,75 sur 1 à 2 semaines pour les génisses de race laitière, puis chute de $5,5 \mathrm{~g} / \mathrm{semaine}$ pendant 3 semaines;

- $+1 \mathrm{~g}$ de $\mathrm{MS} / \mathrm{kg} \mathrm{P}^{0.75}$ sur 1 semaine pour les génisses de race à viande, puis chute de $4,7 \mathrm{~g} / \mathrm{semaine}$ pendant $3 \mathrm{se}$ maines.

Les augmentations sont donc observées toujours au stade montaison.

\section{Teneur en matière sèche}

L'effet de la teneur en matière sèche n'est pas, globalement, significatif pour les 3 catégories de bovins. On observe cependant des tendances à la baisse pour les fourrages les plus humides et à la hausse pour ceux les plus secs. Ainsi, lorsque la teneur en matière sèche varie de 1 point, les quantités ingérées varient dans le même sens :

- de $0,99 \mathrm{~g}$ de $M S / k g P^{0,75}$ pour les vaches laitières (NS);

- de 1,45 g pour les génisses de race laitière $(S)$;

- de 1,16 g pour les génisses de race à viande (NS).

Malheureusement, les taux de refus très différents entre ces 2 cas interdisent une analyse plus poussée du phénomène, que nous n'avions pas toutefois l'intention d'étudier ici.

\section{Digestibilité de la matière organique}

L'effet de la digestibilité de la MO n'est pas significatif sur les quantités d'herbe ingé- rées pour les vaches (amplitude de variation de la DMO égale à 15 points) et les génisses de race laitière (amplitude égale à 20 points). II l'est, en revanche, pour les génisses de race à viande :

$$
\begin{gathered}
\mathrm{QIGV}=-20,1+1,393 \mathrm{DMO} \pm 6,4 \\
r=0,652(\mathrm{~S})
\end{gathered}
$$

\section{Facteurs individuels liés aux animaux}

Nous avons analysé la variabilité intralot des quantités d'herbe ingérées. Les résultats figurent dans le Tableau III. Ils sont présentés sans avoir retiré les effets du poids vif (PV) et de la production de lait (PL4), puis en les retirant.

Pour les vaches laitières, le fait de retirer les effets PV et PL4 réduit sensiblement le coefficient de variation des quantités d'herbe ingérées qui passe de 10,3 à $6,6 \%$. Pour l'ensemble des génisses, le fait de retirer l'effet du poids vif fait passer ces coefficients de variation de 13,9 à $8,9 \%$ pour les quantités ingérées non corrigées et même à $8,5 \%$ pour les quantités en $\mathrm{g} / \mathrm{kg}$ $\mathrm{P}^{0,75}$ (Tableau II). Pour ces animaux, nous avons vérifié que l'effet du gain de poids vif était négligeable. La variabilité des quantités ingérées par les génisses de race laitière reste cependant élevée $(9,5 \%)$ par rapport aux vaches.

\section{Comparaison entre les types d'animaux}

Par souci de cohérence, toutes les comparaisons ont été établies sur la base des données exprimées en $\mathrm{g}$ de $\mathrm{MS} / \mathrm{kg} \mathrm{P} \mathrm{P}^{0,75}$, et recalculées pour les vaches laitières selon les indications du paragraphe précédent. 


\section{Vaches laitières}

Les quantités de MS d'herbe ingérées par les vaches laitières sont reliées à celles ingérées par les moutons et à la production laitière interlots :

QIVL = 33,5 + 0,933 QIM + 1,58 PL4 $\pm 5,7$ $R=0,848 \quad n=26$

A partir de cette relation, on obtient des quantités ingérées égales à $136,6 \mathrm{~g} / \mathrm{kg}$ po,75 pour une production de lait de $21 \mathrm{~kg}$ (valeur très proche de 134 , moyenne des résultats réellement obtenus) et $143 \mathrm{~g} / \mathrm{kg}$ $\mathrm{P}^{0,75}$ pour une production de lait de $25 \mathrm{~kg}$, soit $17,3 \mathrm{~kg}$ de MS d'herbe pour une vache de $600 \mathrm{~kg}$, vache standard du système des unités d'encombrement.

De même, les quantités de MS ingérées par les vaches sont liées à celles ingérées par les génisses:

QIVL $=20,8+0,932$ QIGL + 1,54 PL4 $\pm 5,4 \quad R=0,886 \quad n=19$

QIVL $=44,2+0,844$ QIGV + 1,21 PL4 \pm 7 $R=0,816 n=14$

Toutes les liaisons sont donc significatives. Celle concernant les vaches (avec une production moyenne de $21 \mathrm{~kg}$ de lait) et les moutons est portée sur la Figure 1.

\section{Génisses de race laitière}

D'un échantillon d'herbe à l'autre, la quantité ingérée a été moins variable pour les génisses que pour les moutons (coefficients de variation égaux respectivement à 10 et $13 \%$ ), soit de 65 à $115 \mathrm{~g}$ de $\mathrm{MS} / \mathrm{kg}$ $\mathrm{P}^{0,75}$ pour les génisses et de 43 à $87 \mathrm{~g}$ pour les moutons.

Pour les échantillons d'herbe étudiés ici, nous obtenons la relation suivante:

$\mathrm{QIGL}=57,4+0,436 \mathrm{QIM} \pm 6,8 \quad \mathrm{R}=0,472$ (S) $\mathrm{n}=25$ (Fig. 2).

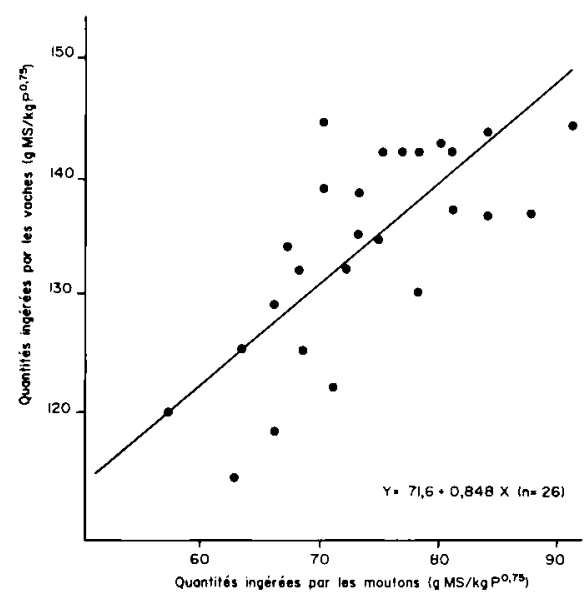

Fig. 1. Relation entre les quantités d'herbe ingérées par les vaches (pour une production de 21 $\mathrm{kg}$ de lait) et les moutons.

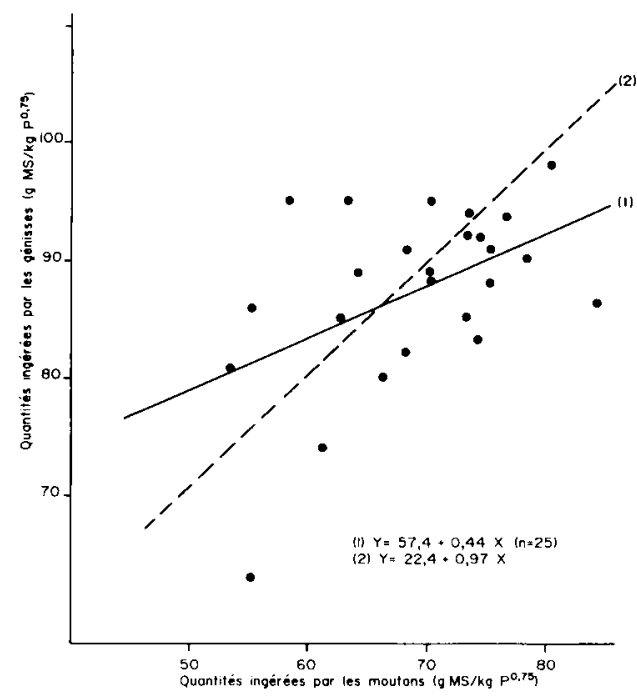

Fig. 2. Relation entre les quantités d'herbe ingérées par les génisses de race laitière et les moutons. 

tif.

L'effet du lieu d'étude n'est pas significa-

\section{Génisses de race à viande}

D'un échantillon d'herbe à l'autre, la quantité ingérée a été plus variable chez les génisses que chez les moutons (coefficients de variation respectivement égaux à 10,8 et $9 \%$ ), soit des quantités allant de 51 à $87 \mathrm{~g}$ de $\mathrm{MS} / \mathrm{kg} \mathrm{P}^{0.75}$ pour les génisses et de 60 à $80 \mathrm{~g}$ pour les moutons recevant les mêmes fourrages.

La relation entre les quantités ingérées par les génisses et les moutons est la suivante :

QIGV $=37+0,559 \mathrm{QIM} \pm 7,2 \quad \mathrm{R}=0,53(\mathrm{~S})$ $n=17$ (Fig. 3)

De même, la relation entre les quantités ingérées par les 2 types de génisse est étroite :

QIGV : - 3,1 + 0,913 QIGL $\pm 3,0 R=0,934$ $n=17$.

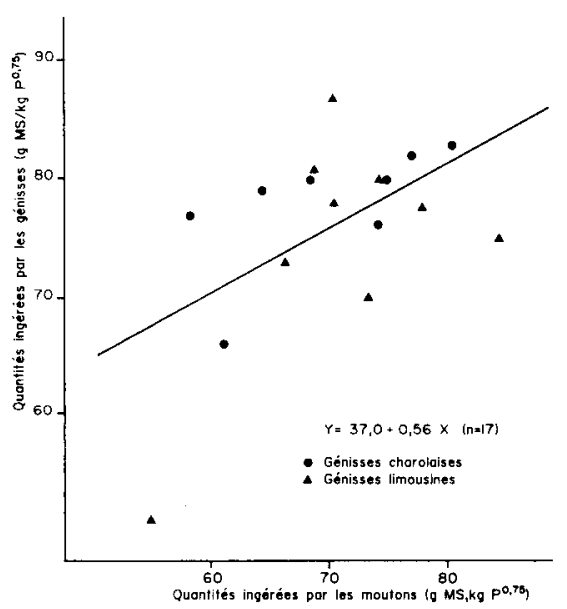

Fig. 3. Relation entre les quantités d'herbe ingérées par les génisses de race à viande et les moutons.

\section{DISCUSSION ET CONCLUSION}

Notre premier objectif était de connaître l'ingestibilité chez les différentes catégories de bovins de l'herbe au stade pâturage, c'est-à-dire l'herbe de référence du système des unités d'encombrement, ingéré à raison de $75 \mathrm{~g}$ de $\mathrm{MS} / \mathrm{kg} \mathrm{P}^{0,75}$ par le mouton standard.

Pour une vache produisant $25 \mathrm{~kg}$ de lait, nous trouvons une valeur de $143 \mathrm{~g}$ de $\mathrm{MS} / \mathrm{kg} \mathrm{P}^{0,75}$ soit $17,3 \mathrm{~kg}$ de MS pour une vache de $600 \mathrm{~kg}$ et, plus précisément 16,8 $\mathrm{kg}$ de MS de fourrage vert lorsque les animaux reçoivent en plus $1,04 \mathrm{~kg}$ de MS de concentré équilibré et $0,87 \mathrm{~kg}$ de $\mathrm{MS}$ de tourteau. Ces quantités d'aliment couvrent les besoins énergétiques d'entretien et de production de $25 \mathrm{~kg}$ de lait (INRA, 1988).

Nous ne disposons pas de résultats bibliographiques concernant des vaches nourries à l'auge, mais au pâturage et, le plus souvent, produisant moins de $20 \mathrm{~kg}$ de lait par jour. Bien que ces derniers résultats ne soient pas comparables aux nôtres, il nous a paru intéressant d'en citer quelques-uns à titre de comparaison.

Pour des vaches produisant respectivement 22,4 et $24 \mathrm{~kg}$ de lait et pesant 536 et $571 \mathrm{~kg}$, Meijs (1981) donne des quantités ingérées de 14,3 et $13,3 \mathrm{~kg}$ de matière organique, avec 0,8 et $1,3 \mathrm{~kg}$ de $\mathrm{MO}$ d'aliment concentré; Hart (1979a et b) donne des valeurs de $13,3 \mathrm{~kg}$ de MO pour des vaches pesant $511 \mathrm{~kg}$, produisant $20 \mathrm{~kg}$ de lait et recevant $0,8 \mathrm{~kg}$ de $\mathrm{MO}$ de concentré. Meijs \& Hoekstra (1984, 2 essais) trouvent des quantités de $M O$ ingérées égales à $14,8 \mathrm{~kg}$ pour les vaches de $577 \mathrm{~kg}$ recevant $0,8 \mathrm{~kg}$ de $\mathrm{MO}$ d'aliment concentré et produisant $24 \mathrm{~kg}$ de lait. La moyenne des résultats de ces 6 essais est de 13,97 kg de MO de fourrage ingéré avec $0,88 \mathrm{~kg}$ de MO d'aliment concentré, soit, en prenant le taux de substitution de 0,5 , l'équivalent de 
$14,4 \mathrm{~kg}$ de $\mathrm{MO}$ de fourrage, c'est-à-dire environ 15,6 de MS pour des vaches de $550 \mathrm{~kg}$ produisant $22,4 \mathrm{~kg}$ de lait. Ces calculs donnent une valeur de $137 \mathrm{~g}$ de MS/ $\mathrm{kg} \mathrm{P} \mathrm{P}^{0,75}$, c'est-à-dire $142 \mathrm{~g}$ environ pour une production de $25 \mathrm{~kg}$ de lait. Cette valeur est identique à celle que nous avons trouvée, mais les animaux ne recevaient pas de tourteau destiné à couvrir "correctement" leurs besoins azotés.

Pour les génisses de race laitière, nos premiers résultats (Dulphy \& MichaletDoreau, 1983) indiquaient, pour un calcul par régression, une valeur de $96 \mathrm{~g}$ de MS/ $\mathrm{kg} \mathrm{P}^{0,75}$. Les résultats supplémentaires obtenus ici conduisent à une valeur plus faible : $90 \mathrm{~g}$ de MS. Pour le système des UE (Dulphy et al., 1987), nous n'avons cependant pas retenu l'ensemble des valeurs obtenues dans ces 2 séries d'essais et nous en avons retiré 5 pour avoir un modèle plus cohérent :

$$
\begin{gathered}
\mathrm{QIGL}=22,4+0,969 \mathrm{QIM} \pm 4,8 \\
r^{2}=0,76 \quad \mathrm{n}=31
\end{gathered}
$$

$\mathrm{Ce}$ modèle donne une ingestibilité de $95 \mathrm{~g}$ de $\mathrm{MS} / \mathrm{kg} \mathrm{P}^{0,75}$ pour l'herbe de référence, correspondant à des quantités de MS ingérées de $8,5 \mathrm{~kg}$ de MS pour des animaux de $400 \mathrm{~kg}$ de poids vif.

Enfin, pour les génisses de race à viande nous trouvons, toujours après calcul par régression, une valeur pour QIM = $75 \mathrm{~g}$ de $\mathrm{MS}$, de $79 \mathrm{~g}$ de $\mathrm{MS} / \mathrm{kg} \mathrm{P} \mathrm{P}^{0,75}$, soit $12 \%$ de moins que pour les animaux de race laitière. La valeur trouvée correspond à des quantités de MS ingérées égales à $7,1 \mathrm{~kg}$ de MS pour des animaux de $400 \mathrm{~kg}$ de poids vif.

Les quantités ingérées d'herbe de référence sont égales, par définition, à la capacité d'ingestion des animaux (Jarrige, 1979). Pour les types de bovin étudiés ici, les valeurs proposées récemment (INRA, 1988) sont respectivement de $17,0 \mathrm{~kg}$ de MS pour des vaches laitières de $600 \mathrm{~kg}$ produisant $25 \mathrm{~kg}$ de lait à $4 \%$ de MG, 8,5 $\mathrm{kg}$ de MS pour des génisses de race laitière, $7,6 \mathrm{~kg}$ de MS pour les génisses Charolaises et $6,7 \mathrm{~kg}$ de MS pour des génisses Limousines, animaux pris tous au poids de $400 \mathrm{~kg}$. Il est évident que les résultats des essais analysés ici ont été largement pris en compte. Dans le système des UE, la capacité d'ingestion des vaches pesant $600 \mathrm{~kg}$ et produisant $25 \mathrm{~kg}$ de lait a été prise à $17 \mathrm{~kg}$ seulement, valeur un peu plus faible que celle trouvée ici, mais qui tient compte des résultats obtenus par ailleurs à Lusignan par I'INRA (Dulphy et al., 1987). La capacité d'ingestion retenue pour les génisses laitières a été celle que nous avons proposée. En revanche, les génisses Charolaises ont une capacité d'ingestion plus faible de $6,6 \%$ dans nos essais, mais les Limousines une capacité plus élevée de 5,6\% par rapport aux valeurs retenues par Troccon et al. (1988).

Notre second objectif était de réactualiser les liaisons établies entre types d'animaux. Etablies par Demarquilly \& Weiss (1971), ces liaisons ne sont pas directement comparables aux nôtres (vaches laitières faibles productrices, concentrés exclus, bœufs et non génisses). Néanmoins, on trouve des coefficients de régression comparables pour les vaches $(0,81$ pour Demarquilly \& Weiss; 0,85 pour nos mesures), ainsi que des écarts types résiduels comparables $(7,1$ contre 6,3 ; l'effet de la production laitière étant pris en compte dans le second cas). En revanche, l'écart type pour les boeufs est très élevé, soit 9,6 , par rapport à 7 pour nos génisses.

Les liaisons obtenues entre vaches et génisses de race laitière, ainsi qu'entre les génisses des 2 types, sont plus étroites que celles entre bovins et moutons. Cela s'explique probablement par le fait que les mécanismes de régulation de l'appétit sont plus proches pour les bovins entre eux, 
qu'entre bovins et ovins. On sait en effet que, souvent, ovins et bovins ne réagissent pas de la même façon aux caractéristiques des fourrages (Dulphy et al., 1984).

Les pentes de 0,4 à 1 trouvées dans les relations entre bovins et ovins confirment les observations de Demarquilly \& Weiss (1971), à savoir que le mouton est un "réactif» plus sensible que le bovin. En effet, lorsque l'ingestibilité d'un fourrage varie de 60 à $80 \mathrm{~g}$ de MS $/ \mathrm{kg} \mathrm{P} \mathrm{P}^{0,75}$ chez le mouton (+ $33 \%$ ), elle varie de 130 à $147 \mathrm{~g}$ chez la vache laitière produisant $25 \mathrm{~kg}$ de lait (+ $13 \%$ seulement en valeur relative). Elle varie par ailleurs de 80,5 à $100 \mathrm{~g}$ pour la génisse de race laitière $(+24 \%)$ avec l'équation retenue plus haut. Ainsi, les variations d'ingestibilité sont comparables en valeur absolue entre les types d'animaux, mais ne le sont pas en valeur relative. Plus l'animal a des besoins élevés, plus il tamponne donc les différences de quantités ingérées, dues ici avant tout à des différences de stade de récolte : à une régulation de l'appétit par des phénomènes digestifs se superpose donc probablement une régulation influencée par les besoins des animaux. La vérification directe de ces observations est cependant difficile, car on ne peut distribuer à des animaux forts producteurs des fourrages tardifs sans un apport élevé d'aliment concentré.

Les coefficients de variation des quantités ingérées intralot n'ont été donnés qu'à titre d'information. On constate cependant qu'ils ne sont pas négligeables pour les génisses ( 8 à $9 \%$ ), mais plus faibles pour les vaches, peut-être parce qu'elles ont pratiquement atteint leur format adulte. Dans la bibliographie, Stakelum (1986a) propose $6 \%$ pour les vaches, Meijs \& Hoekstra (1984) trouvent 5,7 et $4,9 \%$ : toutes ces données sont donc proches de la valeur de $6,6 \%$ que nous avons observée.
Notre but n'était pas d'examiner l'incidence des facteurs liés à la plante comme cela a déjà largement été fait chez les moutons (cf. synthèse de Demarquilly et al., 1981). Signalons cependant la forte influence négative de l'âge de la plante au premier cycle lorsque les épis se développent après un pic d'ingestion très élevé. Cela, joint au fait que la mise en régime des animaux est relativement lente et $\mathrm{li}$ mite l'ingestion en début de premier cycle, explique que, dans nos conditions d'étude, les fourrages de premier cycle et de second cycle ont eu des niveaux d'ingestion comparables, les seconds ayant cependant des valeurs très stables par rapport aux premiers.

L'incidence de l'âge de la plante au premier cycle est classique. II est inutile de la discuter. Cet effet passe par une baisse de la digestibilité et, plus précisément, de la vitesse de digestion (Jarrige et al., 1974). Cependant, cet effet de la digestibilité n'apparaît pas nettement ici à cause de cette faiblesse relative des quantités ingérées en début de premier cycle, alors que la digestibilité est très élevée. Les moutons ont été sensibles à ce phénomène, d'où de bonnes relations entre leurs quantités ingérées et celles ingérées par les bovins. On pourrait expliquer le "déficit" d'ingestion en début de cycle par un encombrement du tube digestif provenant de l'eau contenue dans les fourrages (Vérité \& Journet, 1970), mais la mise en régime sur des fourrages très ingestibles et évoluant progressivement est probablement trop lente, et il serait intéressant d'examiner plus précisément quels sont les facteurs qui limitent l'ingestion au début du premier cycle de végétation.

En définitive, et malgré quelques problèmes à examiner quant aux mécanismes régulant l'ingestion des fourrages verts, on retiendra que les résultats très nombreux de quantité d'herbe ingérée obtenus sur 
moutons par Demarquilly et al. (1981) peuvent être extrapolés aux bovins, moyennant le recours aux relations présentées dans ce texte. Ces relations, calculées pour des animaux conduits simultanément, ne sont en effet pas biaisées par un effet saison auquel les moutons sont très sensibles (Michalet-Doreau \& Gatel, 1983), mais elles ne sont cependant applicables qu'à des animaux comparables à ceux que nous avons utilisés. Au niveau du système des unités d'encombrement, cela n'est pas gênant puisqu'il a été décidé, à la suite des mesures présentées ici, de retenir 2 bovins standard, la vache laitière à $25 \mathrm{~kg}$ de lait et la génisse de race laitière de $400 \mathrm{~kg}$ (Dulphy et al., 1987).

\section{RÉFÉRENCES}

Demarquilly C., Andrieu J. \& Weiss P. (1981) L'ingestibilité des fourrages verts et des foins et sa prévision. In : Prévision de la Valeur Nutritive des Aliments des Ruminants. INRA Publications, route de Saint-Cyr, 78000 Versailles, pp. 155-167

Demarquilly C. \& Weiss P. (1971) Liaisons entre les quantités de matière sèche de fourrage vert ingérées par les moutons et celles ingérées par les bovins. Ann. Zootech. 20, 119134

Dulphy J.P. \& D'Hour (1987) Comparaison des quantités de fourrage vert ingérées entre des vaches laitières, des génisses de race laitière, des génisses de race à viande et des moutons. Reprod. Nutr. Dev. 27, 209-210

Dulphy J.P., Faverdin P., Micol D. \& Bocquier F. (1987) Révision du Système des Unités d'Encombrement (UE). Bull. Tech. CRVZ Theix, INRA 70, 35-48

Dulphy J.P. \& Michalet-Doreau B. (1983) Comportement alimentaire et mérycique d'ovins et de bovins recevant des fourrages verts. Ann. Zootech. 32, 465-474
Dulphy J.P., Michalet-Doreau B. \& Demarquilly C. (1984) Etude comparée des quantités ingérées et du comportement alimentaire et mérycique d'ovins et de bovins recevant des ensilages d'herbe réalisés selon différentes techniques. Ann. Zootech. 33, 291-320

Hart M.L. (1979a) Experiments on the relations between milk production of dairy cows and herbage consumption in pasture. Stencil Landbouwhogeschool. Illrd European Grazing workshop

Hart M.L. (1979b) Comparison of continuous and rotational grazing. Stencil Landbowhogeschool. Illrd European grazing workshop

INRA (1988) Alimentation des ovins, bovins, caprins. INRA Publications, route de Saint-Cyr, 78000 Versailles, $471 \mathrm{p}$.

Jarrige R., Demarquilly C. \& Dulphy J.P. (1974) The voluntary intake of forages. In : Quality of Herbage. Värtodling 28, Uppsala, pp. 98-106

Jarrige R. (1979) Le système des unités d'encombrement pour les bovins. Bull. Techn. CRVZ Theix, INRA 38, 57-79

Jarrige R., Demarquilly C., Dulphy J.P., Hoden A., Robelin J., Beranger C., Geay Y., Journet M., Malterre C., Micol D. \& Petit M. (1986) The INRA «fill unit" system for predicting the voluntary intake of forage-based diets in ruminants. A review. J. Anim. Sci. 63, 1737-1758

Meijs J.A.C. (1981) Herbage intake by grazing dairy cows. In : Agricultural Research Reports 909. Institute for Livestock Feeding and Nutrition Research. "Hoorn" Lelystad, The Netherlands, $264 \mathrm{p}$.

Meijs I.A.C. \& Hoekstra J.A. (1984) Concentrate supplementation of grazing dairy cows. 1 . Effect of concentrate intake and herbage allowance on herbage intake. Grass Forage Sci. 39, 59-66

Michalet-Doreau B. \& Gatel F. (1983) Evolution au cours d'une année des quantités de foin ingérées par des béliers castrés. Ann. Zootech. 32, 459-464

Sarker A.B. \& Holmes W. (1974) The influence of suplementary feeding on the herbage intake and grazing behaviour of dry cows. J. Br. Grassl. Soc. 29, 141-143

Stakelum G. (1986a) Herbage intake of grazing dairy cows. 1. Effect of autumn supplementation with concentration and herbage allowance on herbage intake. Ir. J. Agric. Res. 25, 31-40 
Stakelum G. (1986b) Herbage intake of grazing dairy cows. 2. Effect of herbage allowance, herbage mass and concentrate feeding on the intake of cows grazing primary spring grass. Ir. J. Agric. Res. 25, 41-51

Troccon J.L., Berge P. \& Agabriel J. (1988) Alimentation des veaux et génisses d'élevage. In :
Alimentation des Bovins, Ovins et Caprins. INRA Ed., 471 p.

Vérité R. \& Journet M. (1970) Influence de la teneur en eau et de la déshydratation de l'herbe sur sa valeur alimentaire pour les vaches laitières. Ann. Zootech. 19, 255-268 